\title{
A MAXIMUM RESONANT SET OF POLYOMINO GRAPHS ${ }^{1}$
}

\author{
HePing Zhang ${ }^{2}$ AND Xiangqian Zhou \\ School of Mathematics and Statistics \\ Lanzhou University \\ Lanzhou, Gansu 730000, P.R. China \\ e-mail: zhanghp@lzu.edu.cn \\ zhouxiangqian0502@126.com
}

\begin{abstract}
A polyomino graph $P$ is a connected finite subgraph of the infinite plane grid such that each finite face is surrounded by a regular square of side length one and each edge belongs to at least one square. A dimer covering of $P$ corresponds to a perfect matching. Different dimer coverings can interact via an alternating cycle (or square) with respect to them. A set of disjoint squares of $P$ is a resonant set if $P$ has a perfect matching $M$ so that each one of those squares is $M$-alternating. In this paper, we show that if $K$ is a maximum resonant set of $P$, then $P-K$ has a unique perfect matching. We further prove that the maximum forcing number of a polyomino graph is equal to the cardinality of a maximum resonant set. This confirms a conjecture of $\mathrm{Xu}$ et al. [26]. We also show that if $K$ is a maximal alternating set of $P$, then $P-K$ has a unique perfect matching.
\end{abstract}

Keywords: polyomino graph, dimer problem, perfect matching, resonant set, forcing number, alternating set.

2010 Mathematics Subject Classification: 05C70, 05C90, 92E10.

\section{REFERENCES}

[1] H. Abeledo and G.W. Atkinson, Unimodularity of the Clar number problem, Linear Algebra Appl. 420 (2007) 441-448. doi:10.1016/j.laa.2006.07.026

[2] C. Berge, C.C. Chen, V. Chvátal and C.S. Seow, Combinatorial properties of polyominoes, Combinatorica 1 (1981) 217-224. doi:10.1007/BF02579327

\footnotetext{
${ }^{1}$ This work is supported by NSFC (grant no. 11371180).

${ }^{2}$ Corresponding author.
} 
[3] Z. Che and Z. Chen, Forcing on perfect matchings - A survey, MATCH Commun. Math. Comput. Chem. 66 (2011) 93-136.

[4] E. Clar, The Aromatic Sextet (Wiley, London, 1972).

[5] M.E. Fisher, Statistical mechanics of dimers on a plane lattice, Phys. Rev. 124 (1961) 1664-1672. doi:10.1103/PhysRev.124.1664

[6] E.J. Cockayne, Chessboard domination problems, Discrete Math. 86 (1990) 13-20. doi:10.1016/0012-365X(90)90344-H

[7] C.M. Grinstead, B. Hahne and D. Van Stone, On the queen domination problem, Discrete Math. 86 (1990) 21-26. doi:10.1016/0012-365X(90)90345-I

[8] I. Gutman, S.J. Cyvin, Advances in the Theory of Benzenoid Hydrocarbons (Springer, Berlin, 1990). doi:10.1007/3-540-51505-4

[9] F. Harary, D.J. Klein and T.P. Živkovič, Graphical properties of polyhexes: Perfect matching vector and forcing, J. Math. Chem. 6 (1991) 295-306. doi:10.1007/BF01192587

[10] W.C. Herndon, Resonance energies of aromatic hydrocarbons: Quantitative test of resonance theory, J. Am. Chem. Soc. 95 (1973) 2404-2406. doi:10.1021/ja00788a073

[11] P.W. Kasteleyn, The statistics of dimers on a lattice: I. The number of dimer arrangements on a quadratic lattice, Physica 27 (1961) 1209-1225. doi:10.1016/0031-8914(61)90063-5

[12] X. Ke, A lower bound on the number of elementary components of essentially disconnected generalized polyomino graphs, J. Math. Chem. 50 (2012) 131-140. doi:10.1007/s10910-011-9900-x

[13] D.J. Klein and M. Randić, Innate degree of freedom of a graph, J. Comput. Chem. 8 (1987) $516-521$. doi: $10.1002 /$ jcc. 540080432

[14] W. Li and H. Zhang, Dimer statistics of honeycomb lattices on Klein bottle, Möbius strip and cylinder, Phys. A 391 (2012) 3833-3848. doi:10.1016/j.physa.2012.03.004

[15] S. Liu and J. Ou, On maximal resonance of polyomino graphs, J. Math. Chem. 51 (2013) 603-619. doi:10.1007/s10910-012-0104-9

[16] F. Lu and L. Zhang, Dimers on two types of lattices on the Klein bottle, J. Phys. A 45 (2012) \#49. doi:10.1088/1751-8113/45/49/494012

[17] L. Lovász and M.D. Plummer, Matching Theory (Annals of Discrete Mathematics, Vol. 29, North-Holland, Amsterdam, 1986). 
[18] A. Motoyama and H. Hosoya, King and domino polynomials for polyomino graphs, J. Math. Phys. 18 (1977) 1485-1490.

doi:10.1063/1.523411

[19] L. Pachter and P. Kim, Forcing matchings on square grids, Discrete Math. 190 (1998) 287-294.

doi:10.1016/S0012-365X(97)00266-5

[20] M. Randić, Conjugated circuits and resonance energies of benzenoid hydrocarbons, Chem. Phys. Lett. 38 (1976) 68-70.

doi:10.1016/0009-2614(76)80257-6

[21] M. Randić, Aromaticity and conjugation, J. Am. Chem. Soc. 99 (1977) 444-450. doi:10.1021/ja00444a022

[22] H. Sachs, Perfect matchings in hexagonal systems, Combinatorica 4 (1980) 89-99. doi:10.1007/BF02579161

[23] H. Sachs and H. Zernitz, Remark on the dimer problem, Discrete Appl. Math. 51 (1994) 171-179. doi:10.1016/0166-218X(94)90106-6

[24] K. Salem and H. Abeledo, A maximal alternating set of a hexagonal system, MATCH Commun. Math. Comput. Chem. 55 (2006) 159-176.

[25] S. Wei and X. Ke, Elementary components of essentially disconnected polyomino graphs, J. Math. Chem. 47 (2010) 496-504. doi:10.1007/s10910-009-9589-2

[26] L. Xu, H. Bian and F. Zhang, Maximum forcing number of hexagonal systems, MATCH Commun. Math. Comput. Chem. 70 (2013) 493-500.

[27] W. Yan, Y.-N. Yeh and F. Zhang, Dimer problem on the cylinder and torus, Phys. A 387 (2008) 6069-6078. doi:10.1016/j.physa.2008.06.042

[28] F. Zhang, X. Guo and R. Chen, The connectivity of Z-transformation graphs of perfect matchings of hexagonal systems, Acta Math. Appl. Sin. 4 (1988) 131-135. doi: $10.1007 / \mathrm{bf02006061}$

[29] H. Zhang, The connectivity of Z-transformation graphs of perfect matchings of polyominoes, Discrete Math. 158 (1996) 257-272. doi:10.1016/0012-365X(95)00048-2

[30] H. Zhang and F. Zhang, Perfect matchings of polyomino graphs, Graphs Combin. 13 (1997) 295-304. doi:10.1007/BF03353008

[31] H. Zhang and F. Zhang, Plane elementary bipartite graphs, Discrete Appl. Math. 105 (2000) 291-311. doi:10.1016/S0166-218X(00)00204-3

[32] M. Zheng and R. Chen, A maximal cover of hexagonal systems, Graphs Combin. 1 (1985) 295-298.

doi:10.1007/BF02582955 
Received 9 February 2015

Revised 29 June 2015

Accepted 29 June 2015 